\title{
RDUS
}

Revue de DROIT

UNIVERSITÉ DE SHERBROOKE

Titre : $\quad$ La notion de sujet de droit international ou le défi de sa réforme à l'aune de la responsabilité sociale des entreprises commerciales

Auteur(s): $\quad$ Adeline MICHOUD

Revue : $\quad$ RDUS, 2018, volume 48, numéro 3

Pages: $\quad 243-275$

ISSN : $\quad 0317-9656$

Éditeur : $\quad$ Université de Sherbrooke. Faculté de droit.

URI : $\quad$ http://hdl.handle.net/11143/18407

DOI : $\quad$ https://doi.org/10.17118/11143/18407 
Page vide laissée intentionnellement. 


\section{La notion de sujet de droit international ou le défi de sa réforme à l'aune de la responsabilité sociale des entreprises commerciales}

\section{par Adeline MICHOUD*}

En raison de la montée de la mondialisation, un débat a commencé à émerger pour déterminer si les entreprises multinationales pouvaient être tenues à certaines obligations en matière de droits de la personne en vertu du droit international public et, plus généralement, si les acteurs non étatiques pouvaient être directement soumis aux normes internationales des droits de la personne.

Dans la société et l'économie d'aujourd'hui, la conception du droit international axée sur l'État semble dépassée. En effet, le pouvoir de l'État a considérablement diminué au cours des dernières décennies au profit de celui des entreprises, dont l'importance économique croissante a soulevé des questions quant à la responsabilité juridique des personnes privées en droit international public.

Le présent article traite d'abord de la notion de sujet de droit international et de sa conception originale. L'auteure développera ensuite des arguments en faveur de la reconnaissance des entreprises commerciales en tant que sujets de droit international afin de relever les défis modernes posés par les questions de responsabilité sociale et d'éviter l'impunité des sociétés multinationales.

Titulaire d'un doctorat en droit. Pendant ses études doctorales, l'auteure a travaillé comme assistante d'enseignement et de recherche au Département de droit international privé de l'Université de Genève (2015-2019). Elle est diplômée de plusieurs universités européennes (bachelor en droit anglais, King's College London; master I, Université Paris 1 Panthéon-Sorbonne; maîtrise en droit international, Institut de hautes études internationales et du développement; bachelor et master en droit suisse, Université de Genève). 
With the rise of globalization, a debate has started emerging to determine if transnational corporations could be held to have human rights obligations under international public law and more generally, if non-state actors could directly be subjected to international human rights standards.

In today's society and economy, the state-centric conception of international law appears to be outdated. In fact, state power has substantially declined in the last decades to the profit of corporations, whose growing economic importance has raised questions as to the legal accountability of private persons in international public law.

This article shall discuss the notion of subject of international law and its original conception. The author shall then develop arguments in favour of the recognition of corporate actors as subjects of international law to tackle the modern challenges posed by corporate social responsibility issues and to avoid corporate impunity. 


\section{SOMMAIRE}

I. La notion de sujet en droit international public 253

A. Le concept de personnalité juridique 253

B. La prise en considération progressive des personnes privées en tant que sujets de droit international susceptibles d'avoir des droits et des obligations

II. La nécessité croissante de reconnaître les entreprises comme sujets de droit international public

A. Les réticences relatives à l'extension de la notion de sujet de droit international public.... .262

B. Les arguments en faveur de l'intégration des acteurs de l'entreprise en tant que sujets de droit international. 

Depuis le début des années 90 , une série d'événements tragiques a révélé au public les problèmes générés par certaines pratiques commerciales abusives de plusieurs sociétés multinationales ayant délocalisé leurs activités de production à l'étranger ${ }^{1}$. Les abus de ces entreprises ${ }^{2}$ se matérialisent par des conditions de travail précaires, voire dangereuses, par exemple dans l'industrie textile au Bangladesh ${ }^{3}$ ou le recours généralisé au travail forcé en Thaïlande ${ }^{4}$, ou encore peuvent même aboutir à la pollution de l'environnement et à la destruction des moyens de subsistance de certaines populations locales (illustré notamment par le récent effondrement d'un barrage au Brésil) ${ }^{5}$. On recense ainsi de très

1 On peut notamment citer le conflit de Bougainville en Papouasie-NouvelleGuinée. Pour une analyse plus détaillée de ce conflit relatif à l'exploitation minière dans ce pays, voir : Kristian LASLETT, « State Crime by Proxy: Australia and the Bougainville Conflict», (2012) 52 Brit. J. Criminol. 705; Anthony J. REGAN, "Causes and Course of the Bougainville conflict», (1998) $33 \mathrm{~J}$. Pac. Hist. 269; Herb THOMPSON, «The Economic Causes and Consequences of the Bougainville Crisis », (1991) 17 Resour. Policy 69; Ronald James MAY, « Papua New Guinea's Bougainville Crisis », (1999) 3 Pac. Hist. Rev. 174.

2 À noter que la notion d' « entreprise » telle que nous l'entendons dans le présent article fait référence aux entreprises multinationales à caractère commercial uniquement.

3 Voir notamment Jason BURKE et Saad HAMMADI, « Bangladesh Textile Factory Fire Leaves more than 100 Dead », The Guardian, 25 novembre 2012, en ligne : $<$ https://www.theguardian.com/world/2012/nov/25/bangladesh-textile-factoryfire $>$.

4 Voir notamment: Kate HodAl, Chris Kelly et Felicity LAWRENCE, «Revealed: Asian Slave Labour Producing Prawns for Supermarkets in US, UK ", The Guardian, 10 juin 2014, en ligne : $<$ https://www.theguardian.com/global-development/2014/jun/10/supermarketprawns-thailand-produced-slave-labour $>$.

$5 \quad$ Voir notamment : Zoe SULLIVAN, « Brazil’s Doce River still Foul Eight Months after Dam Collapse », Made for Minds, 5 juillet 2016, en ligne :

$<$ http://www.dw.com/en/brazils-doce-river-still-foul-eight-months-after-damcollapse/a-19375872>; Dom PHILLIPS, « Samarco Dam Collapse: One Year on from Brazil's Worst Environmental Disaster », The Guardian, 15 octobre 2016, en ligne : <https://www.theguardian.com/sustainable-business/2016/oct/15/ samarco-dam-collapse-brazil-worst-environmental-disaster-bhp-billiton-valemining>; voir plus généralement Gregory JACKSON et Julia BARTOSCH, « Corporate Responsibility in Different Varieties of Capitalism: Exploring the Role of National Institutions », Freie Universität Berlin, 2016, en ligne : $<$ https://www.bertelsmann-stiftung.de/fileadmin/files/BSt/Publikationen/Graue Publikationen/Studie_BS_Corporate-Responsibility-in-Different-Varieties-ofCapitalism-Exploring-the-Role-of-National-Institutions_2016.pdf>. 
nombreuses violations des droits de la personne commises par les entreprises multinationales dans les pays en développement ${ }^{6}$.

Le nombre d'entreprises multinationales a connu une croissance exponentielle au cours des 40 dernières années ${ }^{7}$. En 1970, on comptait environ 7000 entreprises multinationales dans le monde : ce nombre est passé à 30000 en 1990, à 63000 en 2000 et à 82000 en $2009^{8}$. Aujourd'hui, on estime à 100000 le nombre d'entreprises multinationales et à plus de 900000 le nombre de leurs filiales dans le monde ${ }^{9}$.

À la faveur de la mondialisation, les entreprises multinationales sont devenues des acteurs clés sur la scène internationale ${ }^{10}$. Leur poids

CONSEIL DES DROITS DE L'HOMME, Report of the Special Representative of the Secretary-General on the Issue of Human Rights and Transnational Corporations and other Business Enterprises - Corporations and Human Rights: A Survey of the Scope and Patterns of Alleged Corporate-related Human Rights Abuse, Doc. N.U. A/HRC/8/5/Add. 2 (23 mai 2008).

7 Voir Ramon TORRENT et Federico LAVOPA, «Strengthening Enforcement of Core Labour Rights: Can a New Investment Agreement Model Help Multinational Corporations Be More Socially Responsible? », dans UNITED NATIONS CONFERENCE ON TRADE AND DEVElOPMENT, Corporate Social Responsibility in Latin America: A Collection of Research Papers From the Virtual Institute Network, Doc. N.U. UNCTAD/DTL/KTCD/2010/2 (février 2010), p. 106-109, en ligne : <https://unctad.org/en/Docs/dtlktcd 20102 en. pdf $>$.

8 United NATIONS CONFERENCE ON TRADE AND DEVELOPMENT, World Investment Report 2009: Transnational Corporations, Agricultural Production and Development, Doc. N.U. UNCTAD/WIR/2009 (26 octobre 2009), en ligne : $<$ http://unctad.org/en/docs/wir2009_en.pdf $>$.

9 Damiano DE FELICE, «Business and Human Rights Indicators to Measure the Corporate Responsibility to Respect: Challenges and Opportunities », (2015) 37 Hum. Rights Q. 511, 517.

10 Joel R. PAUL, «Holding Multinational Corporations Responsible under International Law », (2001) 24 Hastings Int'l \& Comp. L. Rev. 285, 286; Alison Lindsay SHINSATO, «Increasing the Accountability of Transactional Corporations for Environmental Harms: The Petroleum Industry in Nigeria », (2005) 4 Nw. Univ. J. Int'l Hum. Rts. 186, 188; Scott PEGG, "An Emerging Market for the New Millennium: Transnational Corporations and Human Rights », dans Jedrzeg George FRYNAS et Scott PEGG (dir.), Transnational Corporations and Human Rights, Basingstroke, Palgrave Macmillan, 2003, p. 9. 
économique est parfois équivalent, voire supérieur, à celui des États ${ }^{11}$. En effet, une organisation non gouvernementale (ONG) a estimé en 2015 que 69 des plus grandes entités économiques au monde étaient des entreprises privées $^{12}$. Cette montée en puissance des entreprises nécessite de reconsidérer leurs droits et leurs obligations ${ }^{13}$. Au cours des dernières décennies, plusieurs discussions ont été entreprises afin de rechercher des moyens efficaces de réglementer les activités commerciales de ces grandes entreprises, car leurs structures transnationales représentent de nouveaux défis pour la réglementation territoriale à laquelle les États sont généralement habitués ${ }^{14}$.

Être l'objet d'un système juridique implique d'avoir des droits et des devoirs en vertu de ce dernier ${ }^{15}$. En droit interne, les personnes privées telles que les sociétés ont des obligations légales et, à ce titre, peuvent être

11 Phillip I. BlumberG, «Accountability of Multinational Corporations: The Barriers Presented by Concepts of the Corporate Juridical Entity », (2001) 24 Hastings Int'l \& Comp. L. Rev. 297. Voir également CONFÉRENCE DES NATIONS UNIES SUR LE COMMERCE ET LE DÉVELOPPEMENT, Rapport sur l'investissement dans le monde 2019: les zones économiques spéciales, Doc. N.U. UNCTAD/WIR/2019 (2019), en ligne : <https:/unctad.org/fr/Publications Library/wir2019_overview_fr.pdf $>$.

12 Global Justice Now, « $1 \overline{0}$ Biggest Corporations Make more Money than most Countries in the World Combined », 12 septembre 2016, en ligne: $<$ http://www.globaljustice.org.uk/news/2016/sep/12/10-biggest-corporationsmake-more-money-most-countries-world-combined $>$.

13 Mirela V. HRISTOVA, «The Alien Tort Statute: A Vehicle for Implementing the United Nations Guiding Principles for Business and Human Rights and Promoting Corporate Social Responsibility », (2012) 47 U.S.F.L. Rev. 89; Heike FABIG, «The Body Shop and the Ogoni », dans Michael K. ADDo (dir.), Human Rights Standards and The Responsibility of Transnational Corporations, Londres, Kluwer Law International, 1999, p. 309.

14 Sur la question globale du pouvoir exercé par les grandes entreprises, voir : Kean BIRCH, «The Totalitarian Corporation?», (2007) 8-1 Totalitarian Movements and Political Religions 153; Lee DRUTMAN et Charlie CRAY, The People's Business: Controlling Corporations and Restoring Democracy, Oakland (CA), Berrett-Koehler Publishers, 2004; David C. KORTEN, When Corporations Rule the World, West Hartford (CT), Kumarian Press, 1995.

15 Hersch LAUTERPACHT, «The Subjects of International Law », dans Elihu LAUTERPACHT (dir.), International Law, Cambridge, Cambridge University Press, 1970, p. 136-150. 
tenues responsables des violations de ces obligations. Au contraire, le système de droit international public a été conçu à l'origine comme un système interétatique, où les États sont considérés comme les sujets principaux. En vertu des règles actuelles du droit international, les individus, y compris les personnes privées, ne sont pas obligés d'observer certains devoirs, pas plus qu'ils ne peuvent être considérés comme responsables en cas de violations de certaines règles de droit international ${ }^{16}$. En droit international public, la responsabilité de l'État peut effectivement être engagée s'il ne parvient pas à prévenir les abus de ses ressortissants ou des personnes se trouvant sur son territoire. Les conventions de l'Organisation internationale du travail (OIT) prévoient notamment que les États contractants se doivent d'introduire dans leurs systèmes juridiques respectifs des lois nationales en vue d'empêcher les acteurs privés de violer les règles énoncées dans les instruments adoptés par ces États ${ }^{17}$.

Il existe deux approches opposées en droit international : une approche minimaliste et une approche maximaliste. Pour les minimalistes, le droit international public n'est pas le forum approprié pour aborder les questions de responsabilité des entreprises commerciales, le droit international public visant prioritairement à réguler les relations entre États. Au contraire, en ce qui concerne les maximalistes, le droit international public peut et doit être utilisé pour réglementer les actions des personnes privées comme les sociétés ${ }^{18}$.

Pourtant, le préambule de la Déclaration universelle des droits de l'homme, Rés. 217 A (III), Doc. Off. A.G.N.U., $3^{\mathrm{e}}$ sess., suppl. n ${ }^{\circ} 13$, p. 17, Doc. N.U. A/810 (1948), de 1948 prévoyait que les normes relatives aux droits de la personne seraient directement applicables aux acteurs non étatiques, mais ce préambule, comme pour le reste de la Déclaration, semble avoir un effet plus déclaratoire que réellement contraignant.

17 Nous pouvons également citer la Convention sur la lutte contre la corruption d'agents publics étrangers dans les transactions commerciales internationales, OCDE, 17 décembre 1997, en ligne: <http://www.oecd.org/fr/daf/anticorruption/ConvCombatBribery_FR.pdf $>$, et la Convention internationale sur l'élimination de toutes les formes de discrimination raciale, ONU, 21 décembre 1965, en ligne : <https://www.ohchr.org/FR/ProfessionalInterest/Pages/CERD. aspx $>$.

18 Voir les explications fournies par David KINLEY, Justine NoLAN et Natalie 
À l'époque où le système des Nations Unies a été fondé, les États représentaient les uniques acteurs détenant un pouvoir important à l'échelle internationale. De ce fait, les États étaient les seuls à être tenus par le droit international, car ils étaient perçus comme les seuls à pouvoir enfreindre les normes internationales ${ }^{19}$. Or, la mondialisation a contribué à l'émergence de nouveaux acteurs ${ }^{20}$, et l'incidence des multinationales a considérablement augmenté ${ }^{21}$, en ce que ces grandes entreprises peuvent parfois imposer leurs conditions de travail à des pans entiers d'une industrie ou avoir des répercussions substantielles sur le degré de pollution de l'environnement dans lequel des populations vivent ${ }^{22}$.

Ce changement dans les rapports de pouvoir entre États et entreprises nous amène à reconsidérer la répartition de leurs responsabilités respectives. Comme nous l'argumenterons tout au long de notre article, nous pensons qu'il est important de reconnaître l'émergence de sujets de droit international public secondaires, c'est-à-dire des entités possédant une capacité juridique limitée dans certains domaines en fonction de ce que prévoiraient certains traités. La plupart des initiatives adoptées à ce jour ont pris une forme non contraignante, l'une des plus emblématiques ayant été formalisée avec l'adoption des Principes directeurs relatifs aux entreprises et aux droits de l'homme (United Nations Guiding Principles on Business and Human Rights ou UNGP), élaborés par le professeur John Ruggie, représentant spécial du secrétaire

ZERIAL, "The Politics of Corporate Social Responsibility: Reflections on the United Nations Human Rights Norms for Corporations », (2007) 25 C\&SLJ 30, 36.

19 Chris JochNicK, « Confronting the Impunity of Non-State Actors: New Fields for the Promotion of Human Rights », (1999) 21 Hum. Rights Q. 56, 59.

20 Steven R. RATNER, "Corporations and Human Rights: A Theory of Legal Responsibility », (2001-2002) 111 Yale L.J. 443, 468.

21 Commission on Global Governance, Our Global Neighborhood, report, chap. 3: «Promoting Security» (1995), en ligne : <https://www.gdrc.org/u-gov/globalneighbourhood/>. Voir également Christopher May et Louise Amoore, Global Corporate Power, Boulder, Rienner Publisher, 2006, p. 62. 
général des Nations Unies pour les entreprises et les droits de l'homme de 2005 à $2011^{23}$.

La structuration actuelle de la société et de l'économie requiert un changement de statut des sociétés multinationales en droit international public. La mise en œuvre d'obligations de droit contraignant pouvant s'appliquer aux entreprises est progressivement discutée sur la scène internationale. En effet, à la suite d'une proposition élaborée par l'Équateur et l'Afrique du Sud devant le Conseil des droits de l'homme des Nations Unies en septembre 2013, ce dernier a adopté une résolution sur la création d'un groupe de travail intergouvernemental afin de concevoir un instrument juridique contraignant en vue, notamment, de garantir aux victimes d'abus commis par des entreprises un accès à la justice et un droit à réparation ${ }^{24}$. Des négociations sont actuellement en cours aux Nations Unies pour trouver un accord sur l'élaboration de ce traité.

La question de la légitimité d'imposer des obligations de droit international aux entreprises peut être posée. Néanmoins, l'idée selon laquelle le profit devrait être la seule ligne directrice des entreprises doit être repensée ${ }^{25}$. Si l'on considère que les droits de la personne ont une portée universelle et qu'ils servent à protéger les droits fondamentaux des individus, on peut alors estimer que les obligations en la matière s'adressent à tous les acteurs, y compris aux entreprises commerciales ${ }^{26}$.

Nous discuterons donc ci-dessous de la notion de sujet de droit international afin d'évaluer dans quelle mesure elle doit être adaptée pour répondre aux défis actuels posés par les abus perpétrés par certaines

CONSEIL DES DROITS DE L'HOMME DE L'ORGANISATION DES NATIONS UNIES, Principes directeurs relatifs aux entreprises et aux droits de l'homme, Doc N.U. HR/PUB/11/4 (16 juin 2011). CONSEIL DES DROITS DE L'HOMME, Élaboration d'un instrument international juridiquement contraignant sur les sociétés transnationales et autres entreprises et les droits de l'homme, Rés. A/HRC/26/9, 26 $6^{\mathrm{e}}$ session (14 juillet 2014). Norman BowIE, « The Moral Obligations of Multinational Corporations », dans Thomas Donaldson et Thomas DunfeE (dir.), Ethics in Business and Economics, Dartmouth, Dartmouth Publishing, 1997, p. 249. 
entreprises multinationales et remédier aux violations de certains droits de la personne qu'elles génèrent. Dans la première partie de notre article, nous définirons la notion de sujet en droit international et nous traiterons de la suprématie des États en tant que sujets de droit international public. Dans la seconde partie, nous nous pencherons sur l'utilité de reconnaître les entreprises comme sujets de droit international public dans le contexte des abus de responsabilité sociale des entreprises commerciales.

\section{La notion de sujet en droit international public}

\section{A. Le concept de personnalité juridique}

Traditionnellement, toute personne ou toute entité se voit octroyer le statut de sujet d'un ordre juridique si elle réunit trois conditions essentielles. Premièrement, un sujet a des devoirs, ce qui l'oblige à assumer la responsabilité de tout comportement qui serait contraire aux règles du système auquel il appartient. Deuxièmement, un sujet de droit doit être capable de revendiquer certains droits. Troisièmement, un sujet de droit doit posséder la capacité de nouer des relations contractuelles ou d'autres relations juridiques avec d'autres sujets de droit reconnus comme tels dans le système juridique auquel ils appartiennent ${ }^{27}$.

Les systèmes juridiques nationaux comprennent une grande variété de sujets : les personnes physiques (qu'elles soient des ressortissants du même ordre juridique ou qu'elles soient étrangères), les personnes morales (entreprises, associations, ONG, etc.) et aussi les entités étatiques. Les personnes morales font donc partie des principaux sujets des systèmes juridiques nationaux et doivent, de ce fait, en suivre les règles. La plupart des systèmes juridiques prévoient que les individus deviennent sujets de droit de ces systèmes dès la naissance. Quant aux personnes morales, l'État précise en général les conditions qu'elles doivent satisfaire pour se voir reconnaître la personnalité juridique. 
Originellement, le droit international n'admettait que les États au rang de sujet. En effet, le droit international est le droit des Nations, qui s'applique aux États et à leurs relations. Selon la définition classique, le droit international est le droit qui régit les relations interétatiques ${ }^{28}$. Pourtant, l'évolution du monde contemporain a élargi la portée du droit international et a étendu la catégorie de ses sujets aux organisations internationales. Un premier pas vers la prise en considération des parties privées en tant qu'acteurs incontournables du droit international public a été franchi dans l'Affaire de la compétence des tribunaux de Danzig, dans laquelle la Cour permanente de justice internationale (CPJI) a jugé que l'« on ne saurait contester que l'objet même d'un accord international, dans l'intention des parties contractantes, puisse être l'adoption par les parties de règles déterminées créant des droits et des obligations pour les individus et susceptibles d'être appliquées par les tribunaux nationaux ${ }^{29} »$. Ici, la CPJI semblait donc reconnaître que le droit international public pouvait également s'adresser aux personnes privées.

Depuis cet arrêt, le droit international a évolué vers une prise en considération progressive d'autres entités que les seuls États. Par exemple, en 1949, dans l'Affaire des réparations des dommages subis au service des Nations Unies $^{30}$, la Cour internationale de justice (CIJ) a ouvert la voie à une théorie fonctionnelle des sujets juridiques en droit international, car elle a reconnu que les organisations internationales pouvaient être dotées de la personnalité juridique en droit international. La CIJ a ainsi admis la possibilité pour différentes catégories de personnalités juridiques de coexister: «Les sujets de droit, dans un système juridique, ne sont pas nécessairement identiques quant à leur nature ou à l'étendue de leurs droits; et leur nature dépend des besoins de la communauté. Le développement du droit international, au cours de son histoire, a été influencé par les exigences de la vie internationale ${ }^{31}$. »

Rosalyn HigGINS, «Conceptual Thinking about the Individual in International Law », (1978) 24 N. Y. L. Sch. L. Rev. 11, 12.

Affaire de la compétence des tribunaux de Dantzig, C.P.J.I. Recueil des avis consultatif (série B), $\mathrm{n}^{\circ} 15$, p. 17-18 (3 mars 1928).

Réparation des dommages subis au service des Nations Unies, avis consultatif du 11 avril 1949, C.I.J. Recueil 1949, p. 174.

Id., p. 178. 
La justice pénale internationale a aussi participé à l'évolution de la notion de sujet de droit international. Lors du procès de Nuremberg, les juges pénaux ont notamment estimé que les fonctionnaires nazis et leurs collaborateurs pouvaient être tenus responsables de crimes internationaux : « on a fait valoir que le Droit international ne vise que les actes des États souverains et ne prévoit pas de sanctions à l'égard des délinquants individuels [...] Ce sont des hommes et non des entités abstraites qui commettent les crimes dont la répression s'impose, comme sanction du droit international ${ }^{32} »$. Le droit pénal international a donc contribué à reconnaître les personnes privées comme sujets de droit international. La responsabilité individuelle en droit pénal international a été définitivement établie lorsque le Conseil de sécurité de l'Organisation des Nations Unies (ONU) a créé le Tribunal pénal international pour l'ex-Yougoslavie et le Tribunal pénal international pour le Rwanda.

La reconnaissance de la responsabilité individuelle a été plus lente en matière de droits de la personne. Cependant, la formulation employée dans de nombreux instruments internationaux relatifs à cette question indique que les obligations en la matière s'adressent à tous. Par exemple, l'article 4 de la Déclaration universelle des droits de l'homme prévoit ceci : «Nul ne sera tenu en esclavage ni en servitude; l'esclavage et la traite des esclaves sont interdits sous toutes leurs formes. » Cette interdiction de l'esclavage s'adresse donc d'abord aux États, mais aussi aux acteurs privés. À noter que cette déclaration mentionne la responsabilité de "chaque organe de la société33 ». De même, le Pacte international relatif aux droits civils et politiques dispose dans son préambule que "l'individu a des devoirs envers autrui et envers la collectivité à laquelle il appartient et est tenu de s'efforcer de promouvoir et de respecter les droits reconnus dans le présent Pacte $^{34} »$. Ces formulations renforcent donc l'idée que le droit international vise également à réglementer les acteurs privés.

Procès des grands criminels de guerre, Tribunal militaire international de Nuremberg, 14 novembre $1945-1^{\text {er }}$ octobre 1946, p. 125-126, en ligne: $<$ https://www.legal-tools.org/doc/512713/pdf/>.

33 Préc., note 16.

34 Pacte international relatif aux droits civils et politiques, 16 décembre 1966, [1976] 999 R.T.N.U. 171. 
La montée de la mondialisation a brouillé les frontières entre les responsabilités des personnes publiques et des personnes privées. Comme le fait remarquer Jean-Philippe Robé, « la transnationalisation de la société remet en question la souveraineté de l'État; elle conteste le monopole autocertifié que la souveraineté de l'État revendique pour lui-même dans la production de normes, et même la simple capacité des États à produire des normes efficaces ${ }^{35} »$.

$\mathrm{Au}$ cours des dernières années, le rôle que les acteurs privés doivent jouer en droit international a été progressivement reconnu et renforcé. La notion de responsabilité sociale des entreprises illustre bien cette tendance. En effet, ladite notion suppose que les entreprises ont des obligations envers tous les acteurs avec qui elles interagissent et qui sont touchés par leurs activités ${ }^{36}$. Certains auteurs vont même plus loin et affirment que les sociétés multinationales devraient avoir des obligations légales parce qu'elles ont une importance analogue à celle des gouvernements ou aux institutions publiques ${ }^{37}$ en raison de leur taille, de

35 Jean-Philippe RoBÉ, «Multinational Enterprises: The Constitution of a Pluralistic Legal Order», dans Gunther TEUBNER (dir.), Global Law without A State, Aldershot, Dartmouth Publishing Company, 1997, p. 49 (notre traduction).

36 Larry CATA-BACKER, «From Moral Obligation to International Law: Disclosure Systems, Markets and the Regulation of Multinational Corporations ", (2008) 39 Geo. J. Int'l. L. 591, 607; Antonio VIVES, « Corporate Social Responsibility: The Role of Law and Markets and the Case of Developing Countries ", (2008) Chi.Kent. L. Rev. 199, 201: "most people interpret these terms to mean that the corporation has a responsibility toward society, and that the corporation has a responsibility to do something about the problems that affect society; others interpret them to mean that the corporation must take responsibility for its own activities as they affect society ». Voir également les travaux de Jean-Philippe Robé qui argumente en faveur de l'idée que les entreprises multinationales devraient être contrôlées à la manière des États au regard du pouvoir qu'elles ont : Jean-Philippe RoBÉ, «Pour en finir avec Milton Friedman », dans Antoine LYON-CAEN et Quentin URBAN, La crise de l'entreprise et de sa représentation, Dalloz, 2012, p. 11-32; Jean-Philippe RoBÉ, «Les entreprises multinationales, vecteurs d'un nouveau constitutionnalisme », 56 Archives de Philosophie du droit 2013, p. 337-361; Jean-Philippe RoBÉ, «L'au-delà de la doctrine de l'entreprise », Cahiers de droit de l'entreprise, 2013, p. 23-31.

37 Douglas M. BRANSON, «Corporate Social Responsibility Redux », (2001-2002) 76 Tul. L. Rev. 1207, 1216; C.A. Harwell WeLLS, «The Cycles of Corporate 
leur pouvoir économique et de leur influence, ce qui leur confère une nature quasi publique. La notion de responsabilité des entreprises, approuvée par la communauté internationale lors du Sommet mondial pour le développement durable ${ }^{38}$ de 2002 , est une preuve de ce changement de paradigme, car la responsabilité sociale des entreprises implique une attente légitime de voir le secteur privé consacrer certains efforts à l'atteinte des objectifs internationaux de développement durable ${ }^{39}$.

\section{B. La prise en considération progressive des personnes privées en tant que sujets de droit international susceptibles d'avoir des droits et des obligations}

Le droit international public a traditionnellement été perçu comme un système centré sur l'État qui réglemente essentiellement les droits et les obligations des États de même que les relations entre ces derniers ${ }^{40}$. Le but du droit international est donc de favoriser la coopération et la coexistence pacifique à l'échelle interétatique ${ }^{41}$.

Le droit international public a différentes sources ${ }^{42}$, que l'on trouve principalement dans les traités, la coutume internationale et les principes généraux du droit. Composé de directives, de codes de conduite, de normes et de pratiques ${ }^{43}$, le droit «souple» ou «mou» (soft law) n'est

Social Responsibility: an Historical Retrospective for the Twenty-First Century », (2002) 51 U. Kan. L. Rev. 77, 77.

38 Rapport du Sommet mondial pour le développement durable, Doc. N.U. A/CONF.199/20 (26 août au 4 septembre 2002), chap. i, par. 140, et chap. ix, par. 20.

39 Elisa Morgera, Corporate Accountability in International Environmental Law, Oxford, Oxford University Press, 2009, p. 11.

40 Francisco ZAMORA CABOT, Lukas HeCKENDORN URSCHELER et Stephanie DE DYCKER, Implementing the UN Guiding Principles on Business and Human Rights: Private International Law Perspectives, Zurich, Schulthess, 2017, p. 11.

$41 \quad$ Charte des Nations Unies, conclue à San Francisco le 26 juin 1945, préambule.

42 Voir le Statut de la Cour internationale de justice, conclu à San Francisco le 24 octobre 1945, art. 38(1).

43 Fr. ZAMORA CABOt, L. HeCKendorn URSChELER et St. De DyCKeR, préc., note 40, p. 4. 
pas reconnu comme une source de droit international ${ }^{44}$, mais ses principes, qui ne sont pas contraignants, jouent néanmoins un rôle important en droit international public de nos jours, notamment dans le domaine de la responsabilité sociale des entreprises.

En raison des changements induits par la mondialisation, un débat a commencé à émerger pour déterminer si les entreprises multinationales pouvaient être tenues à certaines obligations en matière de droits de la personne en vertu du droit international public ${ }^{45}$ et, plus généralement, si les acteurs non étatiques pouvaient être directement soumis aux normes internationales en matière de droits de la personne ${ }^{46}$. Historiquement, le droit international public a principalement visé à réguler les États. En réalité, selon la conception «classique » du droit international, «seuls les États ont des droits et des devoirs juridiques internationaux : aucune autre entité n'est donc capable de posséder des droits et des obligations

$44 \quad$ Les normes de droit souple ne figurent pas dans la liste de l'article 38 (1) du Statut de la Cour internationale de justice, préc., note 42, qui précise les sources du droit international public.

45 Philip ALSTON, "The "Not-a-Cat" Syndrome: Can the International Human Rights Regime Accommodate Non-State Actors? », dans Philip Alston (dir.), Non-State Actors and Human Rights, Oxford, Oxford University Press, 2005, p. 6; Anne Peters, Lucy Koechlin et Gretta Fenner Zinkernagel, « Non-State Actors as Standard Setters: Framing the Issue in an Interdisciplinary Fashion », dans Anne Peters et autres (dir.), Non-State Actors as Standard Setters, Cambridge, Cambridge University Press, 2009, p. 14; Daphné Josselin et William Wallace, Non-State Actors in World Politics, Basingstoke, Palgrave, 2001, p. 3-4; Mary Ellen O'Connell, «Enhancing the Status of Non-State Actors through a Global War on Terror? », (2005) 43 Colum. J. Transnat'l L. 435, 437.

Sarah JOSEPH, «Taming the Leviathans: Multinational Enterprises and Human Rights », (1999) 46 Neth. Int'l L. Rev. 171, 174; Olivier De SCHUTTER, «The Challenge of Imposing Human Rights on Corporate Actors », dans Olivier DE SCHUTTER (dir.), Transnational Corporations and Human Rights, Oxford et Portland, Hart Publishing, 2006, p. 17-22; Andrew ClaPHAM, Human Rights Obligations of Non-State Actors, Oxford, Oxford University Press, 2006, p. 83; Andrew ClaPHAM, «Human Rights Obligations of Non-State Actors in Conflict Situations », (2006) 88 Int'l. Rev. Red Cross 491, 523; Yaël RonEN, «Human Rights Obligations of Territorial Non-State Actors "), (2013) 46 Cornell Int'l L.J. $21,22-25$. 
juridiques internationaux ${ }^{47} »$. Dans le système économique et l'organisation de la société actuels, cette conception du droit international axée sur l'État semble dépassée. En effet, le pouvoir des États s'est considérablement réduit au cours des dernières décennies au profit de celui des entreprises, dont l'importance économique croissante a soulevé des questions quant à la responsabilité juridique des personnes privées en droit international public ${ }^{48}$.

En droit interne, il est admis depuis longtemps que les personnes morales, telles que les entreprises, ont des obligations juridiques - par exemple en vertu du droit du travail ou du droit de l'environnement - et elles peuvent être tenues responsables des violations de ces obligations. L'instauration de mécanismes judiciaires au niveau national permettant de faire appliquer ces règles de responsabilité des entreprises est par ailleurs indispensable ${ }^{49}$. Le droit interne doit donc complémenter les règles du droit international pour assurer leur bonne mise en œuvre.

$\mathrm{Au}$ regard de l'incidence éventuelle des activités de certaines grandes entreprises, des traités multilatéraux imposent directement des obligations à ces dernières, comme la Convention sur la responsabilité civile dans le domaine de l'énergie nucléaire ${ }^{50}$, la Convention internationale sur la responsabilité civile pour les dommages dus à la

47 Valérie EpPS, International Law, Durham, Carolina Academic Press, 2001, p. 3 (notre traduction).

$48 \quad$ Ce débat est d'autant plus actuel au regard de la montée du recours à l'arbitrage pour remédier aux litiges entre États et investisseurs.

49 Sur cette question, voir: Ivan TCHOTOURIAN et Alexis LANGENFELD, «RSE, droits de l'homme et multinationales : la doctrine du forum non conveniens estelle un obstacle à la judiciarisation du contentieux? », (2019) 2 Revue internationale de droit comparé 503, 503-531; Gwynne SKINNER et autres, «The Third Pillar: Access to Judicial Remedies for Human Rights Violations by Transnational Business ", dans International Corporate Accountability Roundtable, rapport, 2013, p. 63-66.

$50 \quad$ Convention sur la responsabilité civile dans le domaine de l'énergie nucléaire, 29 juillet 1960, telle qu'elle a été modifiée par le Protocole additionnel du 28 janvier 1964 et par le Protocole du 16 novembre 1982, art. 6, en ligne: $<$ https://www.oecd-nea.org/law/nlparis_conv-fr.html $>$. 
pollution par les hydrocarbures ${ }^{51}$ et la Convention sur la responsabilité civile pour les dommages résultant d'activités dangereuses pour l'environnement $t^{52}$, qui imposent toutes des obligations aux entreprises ${ }^{53}$. La prise de conscience croissante de l'incidence potentielle des sociétés multinationales sur les droits de la personne a stimulé l'implantation d'un certain nombre d'initiatives réglementaires depuis la fin du $\mathrm{XX}^{\mathrm{e}}$ siècle.

De nombreuses organisations de la société civile exigent maintenant que des mesures réglementant les activités des entreprises multinationales soient prises au niveau international ${ }^{54}$. Pourtant, à l'heure actuelle, il n'existe aucun instrument en droit international qui impose des obligations contraignantes aux sociétés ${ }^{55}$. En 1998, la Conférence de

Convention internationale sur la responsabilité civile pour les dommages dus à la pollution par les hydrocarbures, 1992, art. 3-4, en ligne: $<$ https://iopcfunds.org/wp-content/uploads/2018/06/Textes-des-Conventions_f. pdf $>$.

Convention sur la responsabilité civile pour les dommages résultant d'activités dangereuses pour l'environnement, Lugano, 21.VI.1993, art. 6, en ligne: $<\mathrm{https}$ ://rm.coe.int/168007c084>.

On peut également citer : Convention de Bâle sur le contrôle des mouvements transfrontières de déchets dangereux et de leur élimination, 5 mai 1992, $\mathrm{n}^{\circ}$ 28911; Convention internationale sur la responsabilité civile pour les dommages dus à la pollution par les hydrocarbures, 1992 (CLC); Convention internationale pour la prévention de la pollution par les navires (MARPOL), 2 octobre 1983.

Jens MARTENS, Corporate Influence on the Business and Human Rights Agenda of the United Nations, Working Paper, MISEREOR, Brot für die Welt and Global Policy Forum 28 (2014), en ligne : <http://ibfan.org/docs/ Corporate_Influence_on_the_Business_and_Human_Rights_Agenda.pdf $>$; The European Union and Transnational Corporations in Latin America: Policies, Instruments and Actors Complicit dans Violations of the Peoples Rights, Permanent Peoples' tribunal, en ligne: <http://permanentpeoplestribunal. org/wp-content/uploads/2010/05/TPP-MADRID-2010-EN.pdf>; voir également Lisa LAPLANTE, «Bringing Effective Remedies Home: The Inter-American Human Rights System, Reparations, and the Duty of Prevention », (2004) 22 Neth. Q. Hum. Rights 347.

Voir John RugGIE, Report of the Special Representative of the Secretary General on the Issue of Human Rights and Transnational Corporations and other Business Enterprises: Guiding Principles on Business and Human Rights: Implementing the United Nations "Protect, Respect and Remedy" Framework, Doc. N.U. A/HRC/17/31 (21 mars 2011), p. 5-6 : 
Rome, qui a adopté le Statut de la Cour pénale internationale, a failli reconnaître la compétence de cette cour pour juger les personnes morales telles que les entreprises, mais la proposition n'a finalement pas recueilli un soutien suffisant ${ }^{56}$.

Cela ne signifie pas pour autant qu'il n'existe aucune règle internationale imposant des obligations aux entreprises en matière de droits de la personne. En ce qui concerne les graves effets préjudiciables que peuvent avoir les opérations transnationales des entreprises multinationales, certains traités multilatéraux imposent directement des obligations aux entreprises, comme c'est le cas des conventions mentionnées plus haut, qui édictent toutes trois des obligations aux entreprises.

Par ailleurs, un projet de traité international est discuté présentement au sein de l'ONU en vue d'établir un instrument juridiquement contraignant pour réglementer les activités des entreprises multinationales dans le contexte du droit international ${ }^{57}$. Voilà qui confirme une certaine volonté de responsabilisation des grandes entreprises. Il reste maintenant à voir si les États parviendront à se mettre

The Guiding Principles' normative contribution lies not in the creation of new international law obligations but in elaborating the implications of existing standards and practices for States and businesses [...] Nothing in these Guiding Principles should be read as creating new international law obligations, or as limiting or undermining any legal obligations a State may have undertaken or be subject to under international law with regard to human rights.

$56 \quad$ Voir plus largement Andrew CLAPHAM, «The Question of Jurisdiction under International Criminal Law over Legal Persons: Lessons from the Rome Conference on an International Criminal Court », dans Menno KAMMINGA et Saman ZIA-ZARIFI (dir.), Liability of Multinational Corporations under International Law, Londres, Kluwer Law International, 2000, p. 139-195.

57 CONSEIL DES DROITS DE L'HOMME DES NATIONS UNIES, Élaboration d'un instrument international juridiquement contraignant sur les sociétés transnationales et autres entreprises et les droits de l'homme, Rés. A/HRC/26/9, $26^{\mathrm{e}}$ session, 14 juillet 2014. 
d'accord sur un ensemble de principes contraignants applicables de manière uniforme au niveau mondial ${ }^{58}$.

\section{La nécessité croissante de reconnaître les entreprises comme sujets de droit international public}

\section{A. Les réticences relatives à l'extension de la notion de sujet de droit international public}

Depuis la fin de la Seconde Guerre mondiale, de nouveaux domaines d'intérêt sont apparus en droit international, où l'on a commencé à prendre en considération les intérêts et les droits des acteurs privés. À l'origine, cette adresse aux acteurs privés était destinée à protéger les individus contre l'intervention injustifiée que l'État peut exercer dans la jouissance des droits individuels ${ }^{59}$, mais les violations des droits de la personne se sont progressivement révélées être également commises par des acteurs privés.

Cependant, les auteurs appartenant à l'école positiviste hésitent encore à considérer les acteurs privés comme des sujets de droit international (plutôt que de simples objets) ${ }^{60}$, malgré l'influence croissante de ces entités ${ }^{61}$ et le déclin considérable du pouvoir étatique ${ }^{62}$. L'une des

Voir également les travaux du PARLEMENT EUROPÉEN, Résolution sur la responsabilité sociale des entreprises : promouvoir les intérêts de la société et ouvrir la voie à une reprise durable et inclusive, 6 février 2013, en ligne : $<$ https:/www.europarl.europa.eu/sides/getDoc.do?reference=P7-TA-2013 0050\&type $=$ TA\&language $=$ FR\&redirect $>$.

August REINISCH, «The Changing International Legal Framework for Dealing with Non-State Actors ", dans Philip ALSTON (dir.), Non-State Actors and Human Rights, Oxford, Oxford University Press, 2005, p. 37.

60 Robert MCCORQUODALE, «Beyond State Sovereignty: The International Legal System and Non-State Participants », (2006) 8 Int'l. L. Rev. Colomb. 103, 122; Robert MC CORQUODALE, «An Inclusive International Legal System », (2004) 17 Leiden J. Int. Law 477; Andrew ClAPHAM, Human Rights Obligations of Non-State Actors, préc., note 46, p. 61; Rosalyn Higgins, Problems and Process: International Law and How We Use It, Oxford, Clarendon Press, 1994, p. 49.

61 Janne E. NIJMAN, «Non-State Actors and the International Rule of Law: Revisiting the Realist Theory of International Legal Personality », (2009) 
principales raisons invoquées est l'objet original du droit international, qui est de réglementer les relations entre les États ${ }^{63}$, tandis que d'autres mettent en doute la légitimité d'élargir le champ des sujets de droit international ${ }^{64}$. Cette position est issue des doctrines allemande et italienne de la fin du XIX ${ }^{\mathrm{e}}$ siècle ou du début du XXe , qui voyaient l'État tel le seul acteur pertinent en droit international ${ }^{65}$. Malgré cela, comme nous en traiterons plus bas, un certain nombre d'auteurs est favorable à l'adaptation de la notion de sujets de droit international à la société actuelle et à l'ouverture de la catégorie de sujets aux acteurs non étatiques ${ }^{66}$.

Amsterdam Center for International Law 1, 4-5; Andrew ClAPHAM, Human Rights Obligations of Non-State Actors, préc., note 46, p. 3.

62 Rafael Domingo, "The Crisis of International Law », (2009), 42 Vand. J. Transnat'l L. 1543, 1551; voir également: Guido ACQUAVIVA, "Subjects of International Law: A Power-based Analysis », (2005) 38 Vand. J. Transnat'l L. 345, 345; Martin VAn CREVEld, The Rise and Decline of the State, Cambridge, Cambridge University Press, 1999, p. 336-340.

63 David KENNEDY, «International Law and the Nineteenth Century: History of an Illusion », (1998) 17 QLR 99, 99.

64 Thomas M. FRANK, The Power of Legitimacy among Nations, Oxford, Oxford University Press, 1990, p. 24; Daniel BODANSKY, « The Concept of Legitimacy in International Law », dans Rudiger WOLFRUM et Volker RÖBEN (dir.), Legitimacy in International Law, New York, Springer, 2008, p. 313-315.

Voir : Jochen VON BERNSTORFF, The Public International Law Theory of Hans Kelsen, Cambridge, Cambridge University Press, 2010, p. 15-43; Janne Elisabeth NiJman, The Concept of International Legal Personality: An Inquiry into the History and Theory of International Law, La Haye, TMC Asser Press, 2004, p. 110-115; Jo Eric Kushal MuRKens, From Empire to Union: Conceptions of German Constitutional Law since 1871, Oxford, Oxford University Press, 2013, p. 19; Reut Yael PAZ, A Gateway between a Distant God and a Cruel World: The Contribution of Jewish German-Speaking Scholars to International Law, Boston, Martinus Nijhoff Publishers, 2013, p. 144-145.

66 Roland PORTMANN, Legal Personality in International Law, Cambridge, Cambridge University Press, 2010, p. 271; Joanna KYRIAKAKIS, «International Legal Personality, Collective Entities and International Crimes », dans Noemi GAL-OR, Cedric Ryngaert et Math NoORTMAnN (dir.), Responsibilities of the Non-State Actor in Armed Conflict and the Market Place: Theoretical and Empirical Findings, Leiden, Brill Nijhoff, 2015, p. 83; Jörg KAMMERHOFER, "Non-State Actors from the Perspective of the Pure Theory of Law », dans Jean D’Aspremont (dir.), Participants in the International Legal System: Multiple Perspectives on Non-State Actors in International Law, Londres, Routledge, 
Le fait que le droit international reste centré sur l'État peut s'expliquer ainsi :

[S]euls les États peuvent créer des règles de droit international par leurs pratiques coutumières et la conclusion de traités. En tant que tels, les États exercent le contrôle ultime pour déterminer le contenu du droit international. Ils sont les artisans du droit international, et c'est donc à eux de choisir d'attribuer certaines responsabilités directement aux entreprises, que ce soit par coutume ou par traité ${ }^{67}$.

Aux États-Unis, le manque de règles internationales claires instaurant la responsabilité des entreprises a été repris par la Cour suprême de ce pays, celle-ci y voyant un indice du rejet de la communauté internationale quant à l'idée de soumettre des entreprises à des obligations de droit international. Cela a été notamment illustré dans l'affaire Kiobel v. Royal Dutch Petroleum $\mathrm{Co}^{68}{ }^{68}$, dans laquelle la Cour suprême a déclaré que « les sources du droit international coutumier ont, à plusieurs reprises, explicitement rejeté l'idée de la responsabilité des personnes morales ${ }^{69} »$. Une argumentation identique a été reprise dans l'affaire Jesner v. Arab Bank en 2018 par la même Cour suprême ${ }^{70}$.

De nombreuses réticences politiques ont freiné les efforts en vue d'établir un régime de responsabilité pour les entreprises en droit

2011, p. 54; Jörg KAMMERHOFER, « The Benefits of the Pure Theory of Law for International Lawyers, or: What Use is Kelsenian Theory », (2006) 12 Int'l Legal Theory 5, 5. Voir également : Jörg KAMMERHOFER, Uncertainty in International Law: A Kelsenian Perspective, Londres, Routledge, 2011; Jean D'AsPREMONT, Formalism and the Sources of International Law: A Theory of the Ascertainment of Legal Rules, Oxford, Oxford University Press, 2011; Jean D'AsPREMONT et Jörg KAMMERHOFER (dir.), International Legal Positivism in a Post-Modern World, Cambridge, Cambridge University Press, 2014. Jennifer ZERK, Multinationals and Corporate Social Responsibility: Limitations and Opportunities in International Law, Cambridge, Cambridge University Press, 2006, p. 262 (notre traduction). 
international ${ }^{71}$. Lors de l'élaboration des principes directeurs de l'ONU, le professeur Ruggie a affirmé qu'aucune responsabilité juridique directe des entreprises ne figurait dans les instruments de droit international tels que la Déclaration universelle des droits de l'homme, les deux pactes des Nations Unies ou encore les conventions de ' $^{\prime} \mathrm{OIT}^{72}$. C'est la raison pour laquelle les Principes directeurs relatifs aux entreprises et aux droits de l'homme distinguent, dans le premier pilier, "le devoir de protéger » de l'État par rapport au second pilier relatif à la «responsabilité de respecter $»$ qui s'adresse aux entreprises ${ }^{73}$.

En doctrine, des auteurs se sont montrés très réticents quant à l'ouverture de la catégorie des sujets de droit international. En effet, certains estiment que les dirigeants utilisant les ressources de l'entreprise pour des activités qui ne sont pas axées sur les bénéfices de l'entreprise violent les droits des actionnaires, dont les intérêts doivent guider toutes les mesures prises par l'entreprise ${ }^{74}$.

71 David KINLEY et Justine NolAN, «Trading and Aiding Human Rights: Corporations in the Global Economy », (2007) 25 Nordisk Tidsskrift for Menneskerettigheter 353, 353; Larry CATA-BACKER, «Multinational Corporations, Transnational Law: The United Nation's Norms on the Responsibilities of Transnational Corporations as Harbinger of Corporate Responsibility in International Law », (2006) 37-2 Colum. Hum. Rts. L. Rev. 287.

72 CONSEIL DES DROITS DE L'HOMME DE L'ORGANISATION DES NATIONS UNIES, Rapport du Représentant spécial du Secrétaire général chargé de la question des droits de l'homme et des sociétés transnationales et autres entreprises, Doc. N.U. A/HRC/4/35, chap. III : «Responsabilité des entreprises pour d'autres violations des droits de l'homme en vertu du droit international ».

73 Voir J. RUGGIE, préc., note 55, principe 11; voir de manière générale : Larry CATÀ-BACKER, «Private Actors and Public Governance beyond the State: The Multinational Corporation, the Financial Stability Board, and the Global Governance Order», (2011) 18 Ind. J. Global Legal Stud. 751; Lara CATABACKER, «Transnational Corporate Constitutionalism: The Emergence of a Constitutional Order for Economic Enterprises », 10 avril 2012, en ligne: $<$ https://ssrn.com/abstract $=203808>$.

74 Thomas M. JONES et Will FELPS, « Shareholder Wealth Maximization and Social Welfare: A Utilitarian Critique », (2013) 23-2 Bus. Ethics Q. 207. Voir, de manière générale, Lynn STOUT, The Shareholder Value Myth: How Putting Shareholders First Harms Investors, Corporations, and the Public, San Francisco, Berrett-Koehler Publishers, 2012. 
Partageant ces conceptions, l'économiste Nieh-hê Hsieh a fait valoir que les entreprises ne devraient pas avoir d'obligations en matière de droits de la personne car, en tant qu'acteurs privés, elles ne sont pas conçues pour assurer le bien-être de la société : «assigner des obligations en matière de droits de la personne aux multinationales revient à leur demander d'adopter une perspective d'impartialité [...] qui semble non seulement trop exigeante mais aussi incompatible avec ce qui est exigé des acteurs privés dans le contexte des activités économiques ${ }^{75} \%$.

Pourtant, on assiste à un changement progressif du rôle reconnu et assigné aux entreprises. À dire vrai, par le passé, celles-ci se souciaient surtout de leur "valeur actionnariale» (shareholders' value) et de la maximisation de leurs profits. Cependant, les entreprises ont petit à petit compris l'importance de prendre en considération la valeur sociale et sociétale (stakeholders' value) de leurs activités relativement aux différentes parties prenantes (les travailleurs, les consommateurs, etc. ${ }^{76}$. Au Royaume-Uni par exemple, la Companies' Act de 2006 a notamment adopté le principe de l'actionnaire éclairé (enlighted shareholder), qui oblige les sociétés à tenir compte d'autres intérêts que ceux des seuls actionnaires dans la mise en œuvre de leurs stratégies commerciales ${ }^{77}$. De même, en mai 2019, la France a modifié l'article 1833 de son code civil pour y intégrer la mention que l'entreprise doit être gérée « en prenant en considération les enjeux sociaux et environnementaux de son activité ${ }^{78} »$. Ce changement dans la loi quant à la raison d'être des entreprises et à leurs obligations est révélateur de la prise de conscience progressive relativement à la nécessité d'imposer aux entreprises des obligations au

Allen Buchanan, The Heart of Human Rights, Oxford, Oxford University Press, 2013, p. 27 (notre traduction).

Henry PETER et Guillaume JACQUEMET, «Corporate Social Responsibility, Sustainable Development et Corporate Governance: quelle corrélation? », (2015) 3 Revue suisse de droit des affaires et du marché financier 170, 172-176. Joan LOUGHREY, Andrew KEAY et Luca CERIONI, «Legal Practitioners, Enlightened Shareholder Value and the Shaping of Corporate Governance », (2008) 8 J. Corp. L. Stud. 79, 86. Des dispositions similaires ont été adoptées en Inde en 2013 avec la Companies Act (adopté le 29 août 2013) : Nitin Pradeep KUMAR, "A Study of CSR Rules under Companies Act 2013 », (2014) Asian J. of Multidiscip. Stud. 142, 145. 
niveau social et environnemental ainsi que de leur rôle à jouer dans la promotion du développement durable.

\section{B. Les arguments en faveur de l'intégration des acteurs de l'entreprise en tant que sujets de droit international}

À l'origine, les entreprises n'ont pas été considérées comme des sujets de droit international. Cette situation tient au fait qu'elles dépendent des États pour obtenir et exercer leurs droits. En effet, une entreprise obtient généralement des droits du fait de ses liens avec un État en particulier (notamment parce que le siège de son administration y est situé ou parce que les personnes à sa tête en ont la nationalité) ${ }^{79}$. La nationalité de l'entreprise lui confère alors certains droits, qui ne sont attribués qu'aux ressortissants de l'État ${ }^{80}$. Par exemple, une entreprise qui possède la nationalité d'un État partie à un traité bilatéral d'investissement (TBI) acquiert des droits en vertu de ce dernier. Par conséquent, la possession d'une nationalité octroie aux entreprises commerciales le droit de bénéficier de la protection diplomatique de l'État dont elles ont la nationalité ${ }^{81}$.

Cependant, les entreprises peuvent également invoquer un certain nombre de règles de droit international, sans nécessairement avoir à demander l'intervention de leur État de nationalité. À n'en pas douter, la protection des individus au niveau international s'est considérablement développée depuis la fin de la Seconde Guerre mondiale. De nos jours, de nombreuses règles internationales (traités ou coutumes) prévoient des droits au bénéfice des individus et des entreprises. Dans le domaine du droit international des droits de la personne, plusieurs traités internationaux comportent des droits pour les personnes privées, ce qui leur permet, en cas de violation par un État, de s'adresser à un mécanisme

James CRAwFORD, Brownlie's Principles of Public International Law, 8 éd., Oxford, Oxford University Press, 2012, p. 528.

$80 \quad$ Id., p. 528-530.

81 Peter T. MuChlinsKi, Multinational Enterprises and the Law, $2^{\mathrm{e}}$ éd., Oxford, Oxford University Press, 2007, p. 705-707. 
de plainte pour obtenir réparation ${ }^{82}$. À titre d'exemple, les entreprises ont pu présenter des requêtes devant la Cour européenne des droits de l'homme en s'appuyant sur la Convention de sauvegarde des droits de l'homme et libertés fondamentales, mieux connue sous le nom de «Convention européenne des droits de l'homme ${ }^{83}$ ».

Les entreprises peuvent aussi intervenir comme amicus curiae dans le cas de poursuites internationales ${ }^{84}$ ou comparaitre devant des groupes spéciaux d'arbitrage international ${ }^{85}$. Néanmoins, la reconnaissance du locus standi devant un tribunal international ou régional ne signifie pas que les sociétés ont la pleine personnalité juridique au niveau international. Étant donné que les acteurs privés jouissent déjà de droits à l'échelle internationale et se sont vu imposer certaines obligations, notamment en vertu du droit pénal international, certains spécialistes en

Le Comité contre la torture du Haut-Commissariat des Nations Unies aux droits de l'homme peut, par exemple, examiner des plaintes individuelles alléguant des violations des droits énoncés dans la Convention contre la torture ou d'autres traitements cruels, inhumains ou dégradants par des États parties qui ont fait la déclaration nécessaire en vertu de son article 22. De même, le Comité pour l'élimination de la discrimination raciale (CERD du haut-commissaire des Nations Unies aux droits de l'homme peut examiner des pétitions individuelles alléguant des violations de la Convention internationale sur l'élimination de toutes les formes de discrimination raciale par des États parties qui ont fait la déclaration nécessaire au titre de son article 14 . Voir notamment Laurence BOISSON DE CHAZOURNES, «Gouvernance et régulation au $21^{\mathrm{e}}$ siècle, quelques propos iconoclastes », dans Laurence BOISSON DE CHAZOURNES et Rostane MEDI, Une société internationale en mutation : quels acteurs pour une nouvelle gouvernance?, Bruxelles, Bruylant, 2005, p. 31. Marius EMBERland, The Human Rights of Companies, Exploring the Structure of ECHR Protection, Oxford, Oxford University Press, 2006, p. 37-41. United States - Import Prohibition of Certain Shrimps and Shrimp products, Affaire $\mathrm{n}^{\circ}$ AB-1998-4, 12 octobre 1998, WT/DS58/AB/R, par. 104 et suiv. (Organe d'appel de l'Organisation mondiale du commerce). Voir généralement Public Citizen's Global Trade Watch, «Case Studies: Investor-State Attacks on Public Interest Policies », 6 mars 2015, en ligne: $<$ https://www.citizen.org/article/case-studies-investor-state-attacks-on-publicinterest-policies/>. 
concluent que le droit international a la capacité d'imposer des obligations horizontales directes aux acteurs privés, en particulier aux entreprises ${ }^{86}$.

Plusieurs auteurs ont plaidé en faveur de la reconnaissance de la qualité de sujet de droit international des entreprises, car ils considèrent qu'elle représenterait une évolution naturelle du droit international moderne ${ }^{87}$. Les auteurs favorables à la reconnaissance des acteurs privés (y compris les entreprises) comme sujets de droit international s'appuient souvent sur le droit international des investissements et la jurisprudence arbitrale : ils invoquent le fait que, si des contrats peuvent être conclus entre un État et une société étrangère, cela suppose que les sociétés ont une personnalité juridique qualifiée ${ }^{88}$.

Dans l'affaire Urbaser S.A. and Consorcio de Aguas Bilbao Bizkaia, Bilbao Biskaia Ur Partzuergoa v. The Argentine Republic ${ }^{89}$, le tribunal arbitral du Centre international pour le règlement des différends relatifs aux investissements (CIRDI) a ouvertement rejeté l'idée que l'investisseur, en tant qu'acteur non étatique, ne puisse être tenu à aucune obligation ${ }^{90}$. Dans ce contexte, le tribunal arbitral a soutenu l'idée que les sociétés, en ce qu'elles bénéficient de plusieurs droits en vertu de traités bilatéraux d'investissement, sont assujetties au droit international et

86

Voir Jakob Katz CoGAN, « The Regulatory Turn in International Law », (2011) 52 Harv. Int'l L.J. 321.

Voir Wolfgang FRIEDMANN, The Changing Structure of International Law, New York, Columbia University Press, 1964, p. 230; voir de manière générale : Wolfgang FrIEDMANN, The Extension of Subjects of International Law beyond the States, La Haye, Académie de La Haye, Recueil des Cours, 1969, p. 121124; David Adedayo IJALAYE, The Extension of Corporate Personality in International Law, New York, Oceana, 1978, p. 221-246; voir, de manière plus générale, Math NoORTMANN et Cédric RYNGAERT (dir.), Non-State Actor Dynamics in International Law: From Law-Takers to Law-Makers, Londres, Routledge, 2016.

Roland PORTMANN, Legal Personality in International Law, Cambridge, Cambridge University Press, 2010, p. 125.

Urbaser S.A. and Consorcio de Aguas Bilbao Bizkaia, Bilbao Biskaia Ur Partzuergoa v. The Argentine Republic, CIRDI, 8 décembre 2016, Affaire $\mathrm{n}^{\circ}$ $\mathrm{ARB} / 07 / 26$.

Id., par. 1194. 
peuvent également avoir des obligations en vertu de ce dernier ${ }^{91}$. Le tribunal arbitral a notamment fait référence à l'article 30 de la Déclaration universelle des droits de l'homme et à l'article 5(1) du Pacte international relatif aux droits économiques, sociaux et culturels pour établir que les parties privées sont pareillement tenues de respecter les droits de la personne ${ }^{92}$. Le tribunal arbitral a donc semblé reconnaître à cette occasion que les personnes privées (y compris les investisseurs et les entreprises) peuvent être considérées comme des sujets de droit international ${ }^{93}$.

Rosalyn Higgins qualifie de chimérique la distinction entre «sujets » et «objets» de droit international ${ }^{94}$. En effet, elle affirme que les entreprises transnationales sont d'importants acteurs dans les systèmes juridiques internationaux et elle soutient que celles-ci devraient, à ce titre, jouer un rôle dans l'élaboration des normes du droit international. Par conséquent, Rosalyn Higgins considère que les entreprises ne peuvent pas ignorer les règles de droit international relatives à la protection des droits de la personne.

L'argument de la morale est souvent avancé par les auteurs en faveur de l'application des normes internationales aux entreprises ${ }^{95}$. Suivant la même tendance, Denis Arnold ajoute que les droits de la personne sont universels, invoquant l'article 30 de la Déclaration universelle des droits de l'homme, ce qui devrait donc entrer parmi les considérations retenues par les entreprises.

Comme l'a indiqué le Conseil des droits de l'homme des Nations Unies, «[1]es sociétés nationales et autres entreprises ont la responsabilité

\footnotetext{
$91 \quad$ Id., par. 1195.

92 Id., par. 1196-1197.

93 Voir également la décision David R. Aven and Others v. Republic of Costa Rica, CIRDI, 18 septembre 2018, Affaire $n^{\circ} \mathrm{UNCT} / 15 / 3$, par. 737-742.

$94 \quad$ R. HigGins, préc., note 60, p. 39-49.

95 Stephen J. Kobrin, «Private Political Authority and Public Responsibility: Transnational Politics, Transnational Firms and Human Rights », (2009) 19-3 Bus. Ethics Q. 349, 351; George G. BRENKERT, Corporate Integrity Accountability, Thousand Oaks, SAGE Publishing, 2004, p. 288-289; Thomas Donaldson, Corporations and Morality, Englewood Cliffs, Prentice-Hall, 1982.
} 
de respecter les droits de l'homme [...] et la capacité de favoriser le bienêtre économique ${ }^{96} »$. Par conséquent, invoquer le fait que les entreprises n'ont pas été créées à l'origine pour entreprendre des activités sociales ne peut en aucun cas légitimer les violations des droits de la personne commises par les entreprises. Même si ces dernières n'ont pas la capacité des États pour exercer un pouvoir sur l'ensemble d'un territoire, elles disposent cependant d'une sphère d'influence leur permettant d'avoir le contrôle sur un nombre important de personnes. Il est donc légitime de leur imposer certains devoirs. La nature privée des sociétés ne doit pas servir de prétexte pour les exonérer de toutes leurs responsabilités ${ }^{97}$.

Les entreprises ont certes principalement une vocation économique. Toutefois, la production et la réalisation de profits ne sauraient écarter des considérations morales majeures, telle l'incidence qu'ont leurs activités sur les populations ou l'environnement ${ }^{98}$. Les systèmes juridiques, $\mathrm{y}$ compris le système de droit international public, se doivent donc d'intégrer ces considérations éthiques et de les promouvoir. La reconnaissance de sujets secondaires de droit international comprenant les entreprises serait une décision efficace à cette fin.

96 Voir le CONSEIL DES DROITS DE L'HOMME, Élaboration d'un instrument international juridiquement contraignant concernant les droits de l'homme pour les sociétés transnationales et autres entreprises, Rés. 26/9, 26 e sess., Doc. N.U. A/HRC/26/L.22/Rev.1, préambule.

97 David BILCHITZ, « Corporate Obligations and a Treaty on Business and Human Rights: A Constitutional Law Model? », dans Surya DEVA et David BILCHITZ (dir.), Building a Treaty on Business and Human Rights: Context and Contours, Cambridge, Cambridge University Press, 2017, p. 205-206; W.M. GREENFIELD, "In the Name of Corporate Social Responsibility », (2004) 47-1 Business Horizons 19, 19-28.

98 Pour une discussion plus poussée sur la question, voir: Ivan TCHOTOURIAN, «Les entreprises ont-elles une responsabilité morale? », Les blogues de Contact de l'Université Laval, 2017, en ligne : $<$ http://www.contact.ulaval.ca/article_blogue/les-entreprises-ont-elles-uneresponsabilite-morale/>; Howard RothMANN BowEN, Social Responsibilities of the Bussinessman, Ann Arbor, Harper \& Brothers, 1953; Claude CHAMPAUD (dir.), L'entreprise dans la société du 21 e siècle, Bruxelles, Larcier, 2013; Kathia MARTIN-CHENUT et René de QUENAUDON (dir.), La RSE saisie par le droit: perspectives interne et internationale, Paris, A. Pedone, 2016. 
Les entreprises ont tout intérêt à prendre en considération les incidences sociales et environnementales de leurs activités. Cet aspect fait partie des revendications croissantes de la société civile à leur endroit ${ }^{99}$. Comme chacun le sait, la réputation d'une entreprise compte parmi ses atouts les plus précieux. La prise en considération des principes de responsabilité sociale par les entreprises les amène donc à gagner en respectabilité et en crédibilité auprès de leurs clients et de leurs investisseurs ${ }^{100}$. Ainsi, l'intégration de la responsabilité sociale permettrait de mieux faire correspondre les pratiques des entreprises aux attentes du grand public.

\section{Conclusion}

À ce jour, les États ont toujours eu le statut de sujets de droit international premiers. Néanmoins, au regard de l'évolution de la société, nous ne pouvons nier qu'il existe également des sujets de droit international secondaires. En ce sens, des obligations juridiques internationales ont été progressivement reconnues à des entités autres que les seuls États.

Comme nous l'avons vu, la CIJ a lancé ce processus, en reconnaissant la personnalité juridique des organisations internationales. Parallèlement, les obligations conférées aux individus, de même que leur accès aux instances internationales dans le domaine pénal, confirment

99

Peter FORSTMOSER, «Corporate Responsibility und Reputation - zwei Schlüsselbegriffe an der Schnittstelle von Recht, Wirtschaft und Gesellschaft », dans Nedim Peter VoGT et autres (dir.), Unternehmen - Transaktion - Recht, Festschrift für Rolf Watter, Zurich, Dike Verlag, 2008, p. 204; Klaus LEISINGER, "Menschenrechte als unternehmerische Verantwortungsdimension », dans Arnd HARDTKE et Annette KLEINFELD, Gesellschaftliche Verantwortung von Unternehmen - Von der Idee der Corporate Social Responsibility zur erforlgreichen Umsetzung, Hamburg, Gabler Verlag, 2010, p. 127.

100 Roman BRETSCHGER, Unternehmen und Menschenrechte - Elemente und Potenzial eines informellen Menschenrechtsschutzes, ZURICH, Schulthess, 2010, p. 55; Hans Caspar VON DER CRONE, «Verantwortlichkeit, Anreize und Reputation in der Corporate Governance der Publikumsgesellschaft », (2000) Zeitschrift für Schweizerisches Recht 239, 271. 
l'idée que les règles de droit international ne sont pas uniquement applicables aux États.

Outre les individus, les entreprises se sont vu progressivement imposer des obligations dans le domaine de l'énergie par l'entremise de différents traités de droit international ${ }^{101}$. Ainsi, les obligations conférées expressément aux États pourraient aussi être imposées à d'autres acteurs privés, dont les entreprises, dans le domaine des droits de la personne. Il ne dépend donc que de la volonté des premiers d'accepter d'accorder aux secondes une capacité juridique internationale limitée par l'intermédiaire des traités internationaux qu'ils adoptent.

Le statut des entreprises en tant que sujets de droit international public susceptibles d'être responsables d'obligations dans le domaine des droits de la personne n'est pas encore clairement établi. Toutefois, il mériterait de l'être. La reconnaissance des sociétés en tant que sujets de droit international représenterait un progrès significatif pour contraindre les entreprises à respecter les droits de la personne et les normes environnementales fixées par les instruments du droit international. Pourtant, une certaine réticence continue à s'exercer à l'encontre de l'actualisation de la notion de sujet de droit international.

Un exemple récent à cet égard se trouve dans le premier projet d'instrument juridiquement contraignant des Nations Unies en vue de réglementer les sociétés multinationales. En effet, le projet, dans sa formulation actuelle, ne prévoit pas l'établissement d'obligations directes pour les sociétés ${ }^{102}$. Cette situation s'explique probablement par une
101
Voir notamment supra, notes 50, 51 et 52 .
Voir OfFICE OF THE High COMMISSIONER FOR HuMAN RigHTS (OHCHR), Legally Binding Instrument to Regulate, in International Human Rights Law, the Activities of Transnational Corporations and other Business Enterprises, $3^{\mathrm{e}}$ sess., Doc. N.U. ZERO DRAFT 16.7.2018 (16 juillet 2018), en ligne : $<$ https://www.ohchr.org/Documents/HRBodies/HRCouncil/WGTransCorp/
Session3/DraftLBI.pdf>. Voir également Charlie HolT, Shira STANTON et Daniel SIMONS, « The Zero Draft Legally Binding Instrument on Business and Human Rights: Small Steps along the Irresistible Path to Corporate Liability ", Greenpeace, [Blogue], 15 septembre 2018, en ligne : $<$ https://www.greenpeace.org/international/story/18581/zerodraft-human-rights- 
absence de volonté politique générale de la part des gouvernements de reconnaître les sociétés comme sujets de droit international public.

Malgré tout, une telle reconnaissance aurait une incidence significative, notamment dans le contexte des jurisprudences nationales. À l'évidence, des tribunaux nationaux invoquent l'inexistence d'obligations au niveau international invocables à l'encontre des entreprises pour justifier leurs décisions de ne pas tenir responsables certains groupes d'entreprises ${ }^{103}$.

Comme l'a souligné à juste titre Rosalyn Higgins, la distinction entre «sujets» et «objets» du droit international relève du mythe juridique. Il semble donc temps d'adapter ce mythe aux défis actuels posés par les activités économiques de la société contemporaine afin d'éviter le règne d'une certaine impunité et ainsi d'assurer une application cohérente des normes internationales à tous les acteurs de la scène internationale, y compris à l'endroit des entreprises multinationales.

Le 28 février 2020, la Cour suprême du Canada, dans l'affaire Nevsun Resources Ltd. c. Araya ${ }^{104}$, a rendu une décision encourageante, porteuse d'espoir pour l'avenir. En effet, la Cour suprême a considéré qu'il n'y avait aucun obstacle «qui écarte la possibilité qu'une réclamation soit présentée contre une société canadienne pour des violations dans un ressort étranger du droit international coutumier, et encore moins du jus cogens ${ }^{105} »$. Dans la même décision, elle a de cette

corporate-accountability/>; Nadia BERNAZ, «The Draft UN Treaty on Business and Human Rights: The Triumph of Realism over Idealism », Business \& Human Rights Resource Centre, en ligne: <https://www.businesshumanrights.org/en/the-draft-un-treaty-on-business-and-human-rights-thetriumph-of-realism-over-idealism>.

103 Voir la décision récente de la Cour suprême des États-Unis : Jesner v. Arab Bank, préc., note 70, p. 16; Kiobel v. Royal Dutch Petroleum Co., préc., note 68, par. 119; voir également John Doe I v. Exxon Mobil Corp., Civil No. 01-1357 (RCL), (D. D.C., 2015), par. 26-27. En France, voir : Cour d'appel de Versailles, $3^{\mathrm{e}}$ chambre, 22 mars 2013, ${ }^{\circ}$ 11/05331, Association France-Palestine Solidarité 'AFPS' c. Société Alstom Transport.

$104 \quad$ Nevsun Resources Ltd. c. Araya, 2020 CSC 5.

$105 \quad I d$. ., par. 122. 
façon reconnu que le droit international coutumier fait automatiquement partie de la common law canadienne ${ }^{106}$, ce qui peut ainsi conduire à la responsabilité de personnes privées sur la base des règles de droit international public ${ }^{107}$. Bien que la Cour suprême ne se soit pas prononcée sur le fond de cette affaire ${ }^{108}$, elle ouvre avec cette décision la voie vers une reconnaissance des personnes privées comme sujets de droit international. L'avenir dira si cette première ouverture sera confirmée par la jurisprudence.

$106 \quad I d$., par. 90 : « le Canada a suivi pendant longtemps la voie conventionnelle de l'incorporation du droit international coutumier au droit interne par application de la doctrine de l'adoption, faisant de celui-ci une partie de la common law canadienne, sauf disposition législative contraire $»$.

$107 I d$., par. 107: «[L]e droit international a tellement pris de l'ampleur [...] qu'il n'existe plus aucune raison valable de restreindre l'application du droit international coutumier aux rapports entre les États. Au cours des 70 dernières années, nous avons assisté à une prolifération de lois relatives aux droits de la personne qui ont transformé le droit international et fait de l'individu une partie intégrante de ce domaine juridique. »

108 Il appartient à présent à la Cour suprême de la Colombie-Britannique de juger le fond de cette affaire. 